\title{
Microwave-assisted synthesis of 1,2,4-triazole-3- carboxamides from esters and amines under neutral conditions
}

\author{
K. R. Jaisankar • K. Kumaran • \\ S. Raja Mohamed Kamil • T. Srinivasan
}

Received: 16 March 2013/ Accepted: 18 June 2013/Published online: 5 July 2013

(C) The Author(s) 2013. This article is published with open access at Springerlink.com

\begin{abstract}
A series of 1,2,4-triazole-3-carboxamides have been prepared from 1,2,4-triazole-3-carboxylates under mild conditions. Efficient synthesis of amides directly from esters and amines is achieved under mild, neutral conditions with liberation of alcohol as by-product. Both primary and secondary aliphatic and aromatic amines can be utilized. This unprecedented, general, environmentally benign reaction proceeds in toluene under microwave conditions. The newly synthesized compounds were characterized by spectral and elemental analyses.
\end{abstract}

Keywords 1,2,4-Triazoles · Hydrazones · Trimethyl aluminium · Microwave assistance

\section{Introduction}

1,2,4-Triazole and its derivatives constitute an important class of organic compounds with diverse agricultural [1], industrial [2] and biological activities [3], including anti-microbial [4, 5], anti-proliferative [6], sedative [7], anticonvulsant [8] and anti-inflammatory actions [9]. Pyridine as a heterocyclic nucleus played a vital role in the development of different medicinal agents and in the field of agrochemicals. This nucleus is present in many products such as drugs, vitamins, food, flavourings, plant dyes, adhesives and herbicides. As part of our program aimed at developing new biologically active compounds, in this work we report the synthesis of some pyridin-3-yl-1,2,4-triazole-3-carboxamides. This report describes a convenient alternative synthesis for triazole carboxamides with improved yields.

K. R. Jaisankar · K. Kumaran $(\bowtie) \cdot$ S. Raja Mohamed Kamil

Department of Chemistry, Islamiah College, Vaniyambadi, Tamil Nadu, India

e-mail: kumaranchem9@gmail.com

T. Srinivasan

Department of Advanced Zoology and Biotechnology, Loyola College, Chennai, India 
In the past few years, use of microwave energy to heat and drive chemical reactions has become increasingly popular in the medicinal chemistry community. This nonclassical heating method has matured from a laboratory curiosity to an established technique that is heavily used in academia and industry. One of the many advantages of using rapid "microwave flash heating" for chemical synthesis is the dramatic reduction in reaction times-from days and hours to minutes and seconds. It was found that the reaction rate was accelerated by about 50 times under isothermal microwave condition compared with conventional isothermal condition, which induces translational and vibrational energy levels and reaction acceleration. The effect of a microwave field on dielectric materials is to induce rapid rotation of the polarized dipoles in the molecules. This generates heat due to friction, simultaneously increasing the probability of contact between the molecules, thus enhancing the reaction rate and reducing the activation energy. As discussed herein, there are good reasons why many pharmaceutical companies are incorporating microwave chemistry into their drug discovery efforts.

\section{Chemistry}

1,2,4-Triazole-3-carboxamides used to be synthesized from 4-arylazo-2-aryl-2oxazolin-5-ones reacting with the appropriate amine. Dyck et al. [10] and Lange et al. [11] prepared 1,2,4-triazole-3-carboxylates and converted these esters to the corresponding amides by saponification, to give carboxylic acid, followed by amidation. The 1,2,4-triazole-3-carboxamides 5a-h described herein were prepared using a different synthetic pathway as outlined in Scheme 1. Diazotization of commercially available 3-aminopyridine in presence of hydrochloric acid gave the diazonium salt, which was directly coupled with ethyl 2-chloroacetoacetate to afford the oxobutanoate 2 [12]. Subsequent treatment with ammonia led to the amine 3. Cyclization of $\mathbf{3}$ with phenylacetyl chloride was achieved by refluxing with toluene. Preparation of the final 1,2,4-triazole-3-carboxamides 5a-h was achieved by adopting a simple one-pot procedure.

In fact, the commonest procedure to prepare carboxamides of heterocycles involves three classical steps: hydrolysis of the ester to acid, followed by acid chloride formation, and finally reaction with the appropriate amine to give the corresponding carboxamide. Conversion of ethyl esters to carboxamides under mild reaction conditions via organoaluminium reagents has been described by Benderly and Stavchansky [13]. Applying this procedure to our 1,2,4-triazole series, the 1,2, 4-triazole-3-carboxamides $\mathbf{5 a}-\mathbf{h}$ were obtained in good yield in a one-step synthesis by treatment of the triazole carboxylate 4 with 2 equiv. of an aluminium complex which was previously prepared in situ by reacting trimethylaluminium with the corresponding amine. However, trimethylaluminium catches fire spontaneously on exposure to air and releases flammable gases in contact with water during work-up. To avoid these practical difficulties, we introduced an atom-economical, environmentally benign direct synthesis of amides from esters and amines under microwave conditions without any catalyst or metal complex [14-16]. 
<smiles>CCOC(=O)/C(N)=N/Nc1cccnc1</smiles>

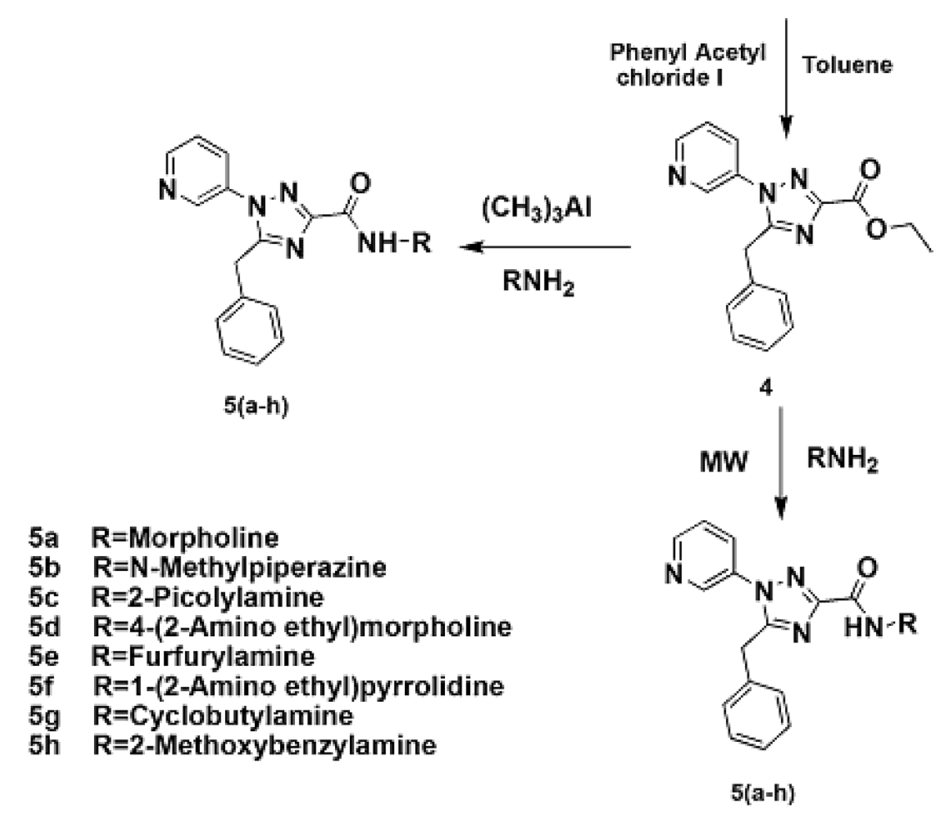

Scheme 1 (a) (i) $\mathrm{NaNO}_{2}$, aq. $\mathrm{HCl}, 0{ }^{\circ} \mathrm{C}$; (ii) ethyl 2-chloroacetoacetate, $\mathrm{NaOAc}, \mathrm{EtOH}, \mathrm{H}_{2} \mathrm{O}, 0{ }^{\circ} \mathrm{C}$; (b) $\mathrm{NH}_{3}$ gas, THF; (c) phenylacetyl chloride, toluene, reflux; (d) [i] corresponding amines for $\mathbf{5 a}-\mathbf{h}$, $\mathrm{Al}(\mathrm{Me})_{3}, \mathrm{~N}_{2}$ atm, toluene, $100{ }^{\circ} \mathrm{C}$; [ii] corresponding amines, toluene, $\mathrm{MW}, 130^{\circ} \mathrm{C}$

Table 1 Conventional heating versus microwave irradiation for amidation reaction

\begin{tabular}{|c|c|c|c|c|c|c|c|}
\hline \multirow[t]{2}{*}{ Compound } & \multirow[t]{2}{*}{ Product } & \multicolumn{3}{|c|}{ Conventional method } & \multicolumn{3}{|c|}{ Microwave irradiation } \\
\hline & & $\begin{array}{l}\text { Time } \\
\text { (h) }\end{array}$ & $\begin{array}{l}\text { Temp. } \\
\left({ }^{\circ} \mathrm{C}\right)\end{array}$ & $\begin{array}{l}\text { Yield } \\
(\%)\end{array}$ & $\begin{array}{l}\text { Time } \\
(\mathrm{min})\end{array}$ & $\begin{array}{l}\text { Temp. } \\
\left({ }^{\circ} \mathrm{C}\right)\end{array}$ & $\begin{array}{l}\text { Yield } \\
(\%)\end{array}$ \\
\hline $5 \mathbf{a}$ & & 12 & 100 & 62 & 30 & 130 & 80 \\
\hline $5 \mathbf{b}$ & & 14 & 100 & 76 & 30 & 130 & 85 \\
\hline
\end{tabular}


Table 1 continued

\begin{tabular}{|c|c|c|c|c|c|c|c|}
\hline \multirow[t]{2}{*}{ Compound } & \multirow[t]{2}{*}{ Product } & \multicolumn{3}{|c|}{ Conventional method } & \multicolumn{3}{|c|}{ Microwave irradiation } \\
\hline & & $\begin{array}{l}\text { Time } \\
\text { (h) }\end{array}$ & $\begin{array}{l}\text { Temp. } \\
\left({ }^{\circ} \mathrm{C}\right)\end{array}$ & $\begin{array}{l}\text { Yield } \\
(\%)\end{array}$ & $\begin{array}{l}\text { Time } \\
(\min )\end{array}$ & $\begin{array}{l}\text { Temp. } \\
\left({ }^{\circ} \mathrm{C}\right)\end{array}$ & $\begin{array}{l}\text { Yield } \\
(\%)\end{array}$ \\
\hline $5 c$ & & 14 & 100 & 75 & 30 & 130 & 90 \\
\hline $5 d$ & & 14 & 100 & 65 & 30 & 130 & 84 \\
\hline $5 e$ & & 16 & 100 & 60 & 30 & 130 & 76 \\
\hline 5f & & 18 & 100 & 50 & 30 & 130 & 65 \\
\hline $5 g$ & & 16 & 100 & 80 & 30 & 130 & 88 \\
\hline $5 \mathrm{~h}$ & & 12 & 100 & 82 & 30 & 130 & 90 \\
\hline
\end{tabular}

\section{Experimental}

All reagents were purchased from Aldrich and used as received. Dry tetrahydrofuran (THF), ethanol and toluene were supplied by Spectrochem. All chemistry was performed under nitrogen atmosphere using standard techniques. Microwave reactions were carried out with a single-mode-cavity Discover microwave 
apparatus. Microwave experiments were performed in a $10-\mathrm{ml}$ sealed tube. All nuclear magnetic resonance (NMR) spectra were measured using a Bruker AMX 400 instrument with 5-mm PABBO BB-1H tubes. ${ }^{1} \mathrm{H}$ and ${ }^{13} \mathrm{C}$ NMR spectra were measured for approximately $0.03 \mathrm{M}$ solutions in $d_{6}$-dimethyl sulphoxide (DMSO) at $400 \mathrm{MHz}$ with tetramethylsilane (TMS) as internal reference. Infrared (IR) spectra were measured on potassium bromide pellets using a PerkinElmer 1600 series Fourier-transform infrared (FTIR) spectrometer. Liquid chromatographymass spectrometry (LC-MS) was carried out using an Agilent 1200 series LC and Micromass zQ spectrometer. Column chromatography was performed using silica gel (230-400 mesh). Combustion analysis was performed on a Costech Elemental Combustion System CHN elemental analyzer.

General procedure for preparation of intermediates

\section{Ethyl 2-chloro-2-[2-(3-pyridyl)hydrazono]acetate (2)}

3-Aminopyridine (25 g, $0.265 \mathrm{~mol})$ was dissolved in $250 \mathrm{ml}$ of $6 \mathrm{~N} \mathrm{HCl}(250 \mathrm{ml})$ solution to give a clear solution and cooled to $0{ }^{\circ} \mathrm{C}$. Sodium nitrite (18.2 g, $\left.0.265 \mathrm{~mol}\right)$ in water $(50 \mathrm{ml})$ was added dropwise to the reaction mass and stirred for $30 \mathrm{~min}$ at the same temperature. Later, ethyl 2-chloroacetoacetate (43 g, $0.265 \mathrm{~mol}$ ) in ethanol $(100 \mathrm{ml})$ was added dropwise for $1 \mathrm{~h}$ at $0{ }^{\circ} \mathrm{C}$. After $30 \mathrm{~min}$, sodium acetate $(65 \mathrm{~g}$, $0.795 \mathrm{~mol})$ in water $(200 \mathrm{ml})$ was added dropwise to the reaction mixture and stirred for $12 \mathrm{~h}$. The precipitated solid was filtered, washed with water, and dried under vacuum to afford pale-yellow crystals of $2(4 \mathrm{~g}, 75 \%)$. M.p. $=124-127{ }^{\circ} \mathrm{C} .{ }^{1} \mathrm{H} \mathrm{NMR}$ (400 MHz, DMSO) $\delta 8.72(\mathrm{~d}, 1 \mathrm{H}), 8.63(\mathrm{~s}, 1 \mathrm{H}), 7.63(\mathrm{~d}, 1 \mathrm{H}), 7.26(\mathrm{~d}, 2 \mathrm{H}), 4.54(\mathrm{q}, 2 \mathrm{H})$, $1.46(\mathrm{t}, 3 \mathrm{H}) ;{ }^{13} \mathrm{CNMR}(400 \mathrm{MHz}, \mathrm{DMSO}) \delta 160.3,156.7,150.89,146.2,134.5,133.3$, 62.2, 32.6, 14.3; LC-MS 229.04 (M+1); Anal. calcd. for $\mathrm{C}_{9} \mathrm{H}_{10} \mathrm{ClN}_{3} \mathrm{O}_{2}$; C: 47.48, H: 4.43, N: 18.46. Found: C: 47.28, H: 4.63, N: 18.30 .

\section{Ethyl 2-amino-2-[2-(3-pyridyl)hydrazono]acetate (3)}

Ammonia gas was bubbled through a solution of $2(10 \mathrm{~g}, 0.044 \mathrm{~mol})$ at $-30{ }^{\circ} \mathrm{C}$ for $30 \mathrm{~min}$ and stirred at room temperature (RT) for $2 \mathrm{~h}$. Thin-layer chromatography (TLC) indicated all $\mathbf{2}$ had been converted to amine 3. The solvent and excess of ammonia were removed under vacuum; the residue was dissolved in $50 \mathrm{ml}$ of dry chloroform and filtered to remove $\mathrm{NH}_{4} \mathrm{Cl}$. The filtrate was evaporated and triturated with diethyl ether $(25 \mathrm{ml})$ to afford pale-brown solid of $3(6.5 \mathrm{~g}, 70 \%)$. M.p. $=85-88{ }^{\circ} \mathrm{C}$; ${ }^{1} \mathrm{H} \mathrm{NMR}$ (400 MHz, DMSO) $\delta 8.71(\mathrm{~m}, 1 \mathrm{H}), 8.59(\mathrm{~s}, 1 \mathrm{H}), 7.61(\mathrm{~d}, 1 \mathrm{H}), 7.38(\mathrm{~d}, 1 \mathrm{H}), 4.65(\mathrm{br}, 2 \mathrm{H})$, 4.32 (q, 2H), 1.34 (t, 3H); ${ }^{13} \mathrm{C}$ NMR (400 MHz, DMSO) $\delta 159.3,154.5,140.2,134.4$, 125.4, 123.0, 116.2, 62.5, 14.1; MS 209.2 (M+1); Anal. $\mathrm{C}_{9} \mathrm{H}_{12} \mathrm{~N}_{4} \mathrm{O}_{2}$. C: 51.92, H: 5.81, $\mathrm{N}: 26.91$. Found: C: 47.98, H: 5.83, N: 26.90.

\section{Ethyl 5-benzyl-1-(pyridin-3-yl)-1H-1,2,4-triazole-3-carboxylate (4)}

Phenylacetyl chloride $(4.4 \mathrm{~g}, 0.0288 \mathrm{~mol})$ was added dropwise to a stirred solution of $3(5 \mathrm{~g}, 0.024 \mathrm{~mol})$ in toluene $(25 \mathrm{ml})$ at $0{ }^{\circ} \mathrm{C}$. The reaction mass was warmed to RT 
and heated to reflux for $12 \mathrm{~h}$. After completion of the reaction, the solvent was evaporated. The residue was dissolved in $\mathrm{CH}_{2} \mathrm{Cl}_{2}(120 \mathrm{ml})$ and washed successively with $1 \mathrm{~N} \mathrm{HCl}, 10 \% \mathrm{NaHCO}_{3}$ and brine. The organic layer was dried over anhydrous $\mathrm{Na}_{2} \mathrm{SO}_{4}$ and evaporated under reduced pressure. The residue was purified on silica gel (hexane/EtOAc 6:4) to give the desired product 4 as a brown gummy solid $(0.31 \mathrm{~g}$, $36 \%)$. M.p. $=160-166{ }^{\circ} \mathrm{C} ;{ }^{1} \mathrm{H}$ NMR $\left(400 \mathrm{MHz}, \mathrm{DMSO}-d_{6}\right) \delta 8.71(\mathrm{~d}, 1 \mathrm{H}), 8.59(\mathrm{~S}$, $1 \mathrm{H}), 7.61(\mathrm{~d}, 1 \mathrm{H}), 7.38(\mathrm{dd}, 2 \mathrm{H}), 7.26(\mathrm{~d}, 2 \mathrm{H}), 7.04(\mathrm{~d}, 2 \mathrm{H}), 4.52(\mathrm{dd}, 2 \mathrm{H}), 4.24(\mathrm{~S}, 2 \mathrm{H})$, $1.46(\mathrm{t}, 3 \mathrm{H}) ;{ }^{13} \mathrm{C}$ NMR (400 MHz, DMSO- $\left.d_{6}\right) \delta 150.89,146.24,133.5,129.38$, 128.34, 127.45, 123.8, 62.23, 32.63, 14.31; LC-MS: $315.4(\mathrm{M}+1)$. Anal.calcd. for $\mathrm{C}_{17} \mathrm{H}_{16} \mathrm{~N}_{4} \mathrm{O}_{2} \mathrm{C}: 66.22, \mathrm{H}: 5.23, \mathrm{~N}: 18.17$, O: 10.38. Found: C: 66.20, H: 5.26, N: 18.20.

\section{General procedure for synthesis of 1,2,4-triazole-3-carboxamides 5a-h}

Conventional method: To a solution of the corresponding amine (1.1 eq.) in dry toluene $(5 \mathrm{ml})$ was added a solution of $\mathrm{Al}(\mathrm{Me})_{3}$ in toluene $\left(2 \mathrm{M}, 2.0\right.$ eq.) under $\mathrm{N}_{2}$ atmosphere. The reaction mixture was stirred at RT for $1 \mathrm{~h}$. A solution of 4 (1 eq.) in dry toluene $(6 \mathrm{ml})$ was then added dropwise. The mixture was heated to $90{ }^{\circ} \mathrm{C}$ during the respective time, then carefully poured onto $1 \mathrm{~N} \mathrm{HCl}(30 \mathrm{ml})$. The biphasic solution was heated to $40{ }^{\circ} \mathrm{C}$ for $30 \mathrm{~min}$ and cooled to RT. The organic layer was separated, dried over anhydrous $\mathrm{Na}_{2} \mathrm{SO}_{4}$ and evaporated. The crude product was purified by column chromatography.

Microwave irradiation: To a solution of the corresponding amine (1.1 eq.) in dry toluene $(5 \mathrm{ml})$ was added 4 ( 1 eq.), followed by heating to $130{ }^{\circ} \mathrm{C}$ under microwave conditions for $30 \mathrm{~min}$. The solvent was removed under vacuum, and the crude purified by column chromatography.

\section{[5-Benzyl-1-(pyridin-3-yl)-1H-1,2,4-triazol-3-yl](morpholino)methanone (5a)}

Conventional method: Compound 5a was prepared from 4 (250 mg, $0.8 \mathrm{mmol})$, morpholine $(83 \mathrm{mg}, 0.9 \mathrm{mmol})$ and $\mathrm{Al}(\mathrm{Me})_{3}(3.2 \mathrm{ml}, 1.6 \mathrm{mmol})$; reaction time: $12 \mathrm{~h}$; purified by flash chromatography [pet.ether/EtOAc (4:6)]; yield: $175 \mathrm{mg}$ (62\%) as brown solid.

Microwave irradiation: Compound 5a was prepared from 4 (250 mg, $0.8 \mathrm{mmol})$ and morpholine ( $83 \mathrm{mg}, 0.9 \mathrm{mmol}$ ), $30 \mathrm{~min}$; purified by flash column chromatography [pet. ether/EtOAc (4:6)]; yield: $226 \mathrm{mg}(80 \%)$ as brown solid. M.p. $=195-198{ }^{\circ} \mathrm{C}$; ${ }^{1} \mathrm{H}$ NMR (400 MHz, DMSO) $\delta 8.72(\mathrm{~d}, 1 \mathrm{H}), 8.63(\mathrm{~s}, 1 \mathrm{H}), 7.63(\mathrm{~d}, 1 \mathrm{H}), 7.40(\mathrm{~m}, 1 \mathrm{H})$, $7.26(\mathrm{~m}, 3 \mathrm{H}), 7.09$ (d, 2H), 4.20 (s, 2H), $3.96(\mathrm{t}, 2 \mathrm{H}), 3.85(\mathrm{~m}, 2 \mathrm{H}), 3.80(\mathrm{~m}, 2 \mathrm{H}), 3.74$ $(\mathrm{m}, 2 \mathrm{H}) ;{ }^{13} \mathrm{C}$ NMR (400 MHz, DMSO) $\delta$ 150.6, 146.0, 132.7, 128.9, 128.4, 127.4, 123.8, 67.0, 66.8, 47.5, 42.9, 32.5. LC-MS: $350.3(\mathrm{M}+1)$. Anal. calcd. for $\mathrm{C}_{19} \mathrm{H}_{19} \mathrm{~N}_{5} \mathrm{O}_{2}$; C: 65.32, H: 5.48, N: 20.04. Found: C: 65.30, H: 5.50, N: 20.07.

\section{[5-Benzyl-1-(pyridin-3-yl)-1H-1,2,4-triazol-3-yl](4-methylpiperazin-1-} $y l)$ methanone $(\mathbf{5 b})$

Conventional method: Compound 5b was prepared from 4 (250 mg, $0.8 \mathrm{mmol})$, $\mathrm{N}$-methylpiperazine $(90 \mathrm{mg}, 0.9 \mathrm{mmol})$ and $\mathrm{Al}(\mathrm{Me})_{3}(3.2 \mathrm{ml}, 1.6 \mathrm{mmol})$; reaction 
time: $14 \mathrm{~h}$; purified by flash column chromatography [pet. ether/EtOAc (4:6)]; yield: $214 \mathrm{mg}(76 \%)$ as off-white solid.

Microwave irradiation: Compound 5b was prepared from 4 (250 mg, $0.8 \mathrm{mmol})$ and $\mathrm{N}$-methylpiperazine ( $90 \mathrm{mg}, 0.9 \mathrm{mmol}$ ), $30 \mathrm{~min}$; purified by flash column chromatography [pet. ether/EtOAc (4:6)]; yield: $249 \mathrm{mg}(85 \%)$ as off-white solid. M.p. $=115-118{ }^{\circ} \mathrm{C} ;{ }^{1} \mathrm{H}$ NMR (400 MHz, DMSO) $\delta 8.71(\mathrm{~d}, 1 \mathrm{H}), 8.63(\mathrm{~s}, 1 \mathrm{H}), 7.65$ $(\mathrm{d}, 1 \mathrm{H}), 7.36(\mathrm{~d}, \mathrm{~J}=8.7 \mathrm{~Hz}, 2 \mathrm{H}), 7.26(\mathrm{~d}, 2 \mathrm{H}), 7.01(\mathrm{~d}, 2 \mathrm{H}), 4.22(\mathrm{~S}, 2 \mathrm{H}), 3.92(\mathrm{t}$, 4H) $2.48(\mathrm{t}, 4 \mathrm{H}), 2.33(\mathrm{~s}, 3 \mathrm{H}) ;{ }^{13} \mathrm{C}$ NMR (400 MHz, DMSO) $\delta 160.0,157.5,155.2$, $150.5,146.0,134.8,133.6,132.7,130.0,128.9$, 128.3, 127.4, 123.8, 55.3, 54.6, 46.9, 45.9, 42.4, 32.5. LC-MS (ESI) $m / z: 363.1(\mathrm{M}+1)$. Anal. calcd. for $\mathrm{C}_{20} \mathrm{H}_{22} \mathrm{~N}_{6} \mathrm{O}$; C: 66.28; H: 6.12; N: 23.19. Found: C: 66.30, H: 6.15, N: 23.20.

\section{5-Benzyl-N-(pyridin-2-yl)methyl)-1-(pyridin-3-yl)-1H-1,2,4-triazole-3-carboxamide} $(5 c)$

Conventional method: Compound 5c was prepared from 4 (250 mg, $0.8 \mathrm{mmol})$, 2-picolylamine (97 $\mathrm{mg}, 0.9 \mathrm{mmol})$ and $\mathrm{Al}(\mathrm{Me})_{3}(3.2 \mathrm{ml}, 1.6 \mathrm{mmol})$; reaction time: $14 \mathrm{~h}$; purified by flash chromatography; yield: $226 \mathrm{mg}$ (75\%), $270 \mathrm{mg}$ (90\%) as brown solid.

Microwave irradiation: Compound 5c was prepared from 4 (250 mg, $0.8 \mathrm{mmol})$ and 2-picolylamine (97 mg, $0.9 \mathrm{mmol}), 30 \mathrm{~min}$; column purification [pet. ether/ EtOAc(5:5)]; yield: $270 \mathrm{mg}(90 \%)$ as brown solid. M.p. $=145-148{ }^{\circ} \mathrm{C} ;{ }^{1} \mathrm{H} \mathrm{NMR}$ (400 MHz, DMSO) $\delta 8.71(\mathrm{~d}, 1 \mathrm{H}), 8.63(\mathrm{~s}, 1 \mathrm{H}), 8.59(\mathrm{~d}, 1 \mathrm{H}), 8.24(\mathrm{br}, 1 \mathrm{H}), 7.67$ (m, $2 \mathrm{H}), 7.39(\mathrm{~m}, 2 \mathrm{H}), 7.21-7.31(\mathrm{~m}, 4 \mathrm{H}), 7.1(\mathrm{~d}, 2 \mathrm{H}), 4.82(\mathrm{~d}, 2 \mathrm{H}), 4.22(\mathrm{~S}, 2 \mathrm{H}) ;{ }^{13} \mathrm{C}$ NMR (400 MHz, DMSO) $\delta$ 150.6, 149.1, 146.0, 136.5,132.8, 128.9, 128.3, 127.4, 123.8, 122.5, 122.3, 44.4, 32.6. LC-MS (ESI) $\mathrm{m} / \mathrm{z}: 371.4(\mathrm{M}+1)$. Anal. calcd. for $\mathrm{C}_{21} \mathrm{H}_{18} \mathrm{~N}_{6} \mathrm{O}$; C: 68.09; H: 4.90; N: 22.69. Found: C: 68.01; H: 4.30; N: 22.30 .

\section{5-Benzyl-N-(morpholinoethyl)-1-(pyridin-3-yl)-1H-1,2,4-triazole-3-carboxamide $(5 d)$}

Conventional method: Compound 5d was prepared from 4 (250 mg, $0.8 \mathrm{mmol})$, 2-aminoethyl morpholine $(117 \mathrm{mg}, 0.9 \mathrm{mmol})$ and $\mathrm{Al}(\mathrm{Me})_{3}(3.2 \mathrm{ml}, 1.6 \mathrm{mmol})$; reaction time: $14 \mathrm{~h}$; purified by flash chromatography [pet. ether/EtOAc (5:5)]; yield: $206 \mathrm{mg}(65 \%)$ as off-white solid.

Microwave irradiation: Compound 5d was prepared from 4 (250 mg, $0.8 \mathrm{mmol}$ ) and 2-aminoethyl morpholine $(117 \mathrm{mg}, 0.9 \mathrm{mmol}), 30 \mathrm{~min}$; purified by flash chromatography [pet. ether/EtOAc (1:1)]; yield: $267 \mathrm{mg}(84 \%)$ as brown solid. M.p $=115-118{ }^{\circ} \mathrm{C} ;{ }^{1} \mathrm{H}$ NMR (400 MHz, DMSO) $\delta 8.71(\mathrm{~d}, \mathrm{~J}=5 \mathrm{~Hz}, 1 \mathrm{H}), 8.63(\mathrm{~m}$, 2H), 7.68 (d, J=8 Hz, 1H), $7.60(\mathrm{br}, 1 \mathrm{H}), 7.41(\mathrm{~m}, 1 \mathrm{H}), 7.21-7.31(\mathrm{~m}, 3 \mathrm{H}), 7.1$ (d, $\mathrm{J}=5.6 \mathrm{~Hz}, 2 \mathrm{H}), 4.22(\mathrm{~s}, 2 \mathrm{H}), 3.72(\mathrm{~m}, 4 \mathrm{H}), 3.62(\mathrm{~m}, 2 \mathrm{H}), 2.63(\mathrm{~m}, 2 \mathrm{H}), 2.53(\mathrm{~m}$, $4 \mathrm{H}) ;{ }^{13} \mathrm{C}$ NMR (400 MHz, DMSO) $\delta$ 158.9, 155.9, 150.6, 146.0, 134.7, 133.6, 132.8, 129.5, 128.9, 128.3, 66.9, 56.9, 53.3, 35.8, 32.6. LCMS : $393.2(\mathrm{M}+1)$. Anal. Calcd for $\mathrm{C}_{21} \mathrm{H}_{24} \mathrm{~N}_{6} \mathrm{O}_{2}$; C: 64.27; H: 6.16; N: 21.41. Found: C: 64.01; H: 6.30; N: 21.30. 
5-Benzyl-N-[(furan-2-yl)methyl]-1-(pyridin-3-yl)-1H-1,2,4-triazole-3-carboxamide $(5 e)$

Conventional method: Compound 5e was prepared from 4 (250 mg, $0.8 \mathrm{mmol})$, furfurylamine $(87 \mathrm{mg}, 0.9 \mathrm{mmol})$ and $\mathrm{Al}(\mathrm{Me})_{3}(3.2 \mathrm{ml}, 1.6 \mathrm{mmol})$; reaction time: $16 \mathrm{~h}$; purified by flash chromatography [pet. ether/EtOAc (5:5)]; yield: $174 \mathrm{mg}$ (60\%): $152 \mathrm{mg}$ as brown viscous liquid.

Microwave irradiation: Compound 5e was prepared from 4 (250 mg, $0.8 \mathrm{mmol})$ and furfurylamine $(87 \mathrm{mg}, 0.9 \mathrm{mmol}), 30 \mathrm{~min}$; purified by flash chromatography [pet. ether/EtOAc (1:1)]; yield: $221 \mathrm{mg}(76 \%)$ as viscous brown liquid. ${ }^{1} \mathrm{H}$ NMR (400 MHz, DMSO) $\delta 8.71(\mathrm{~d}, 1 \mathrm{H}), 8.63(\mathrm{~s}, 1 \mathrm{H}), 7.68(\mathrm{~d}, 1 \mathrm{H}), 7.42(\mathrm{br}, 1 \mathrm{H}), 7.38(\mathrm{~m}$, 2H), $7.31(\mathrm{~m}, 3 \mathrm{H}), 7.09(\mathrm{~d}, 2 \mathrm{H}), 6.33(\mathrm{~d}, 2 \mathrm{H}), 4.68(\mathrm{~s}, 2 \mathrm{H}), 4.19(\mathrm{~s}, 2 \mathrm{H}) ;{ }^{13} \mathrm{C} \mathrm{NMR}$ (400 MHz, DMSO) $\delta$ 150.7, 146.0, 142.4, 132.8, 129.0, 128.3, 127.4, 123.8, 110.5, 107.9, 36.3, 32.5. LC-MS (ESI) $m / z: 360.3(\mathrm{M}+1)$. Anal. calcd. for $\mathrm{C}_{20} \mathrm{H}_{17} \mathrm{~N}_{5} \mathrm{O}_{2}$; C: 66.84; H: 4.77; N: 19.49; Found: C: 66.44, H: 4.97, N: 19.69.

\section{5-Benzyl-1-(pyridin-3-yl)-N-[2-(pyrrolidin-1-yl)ethyl]-1H-1,2,4-triazole-3- carboxamide $(\mathbf{5 f})$}

Conventional method: Compound $5 \mathbf{f}$ was prepared from 4 (250 mg, $0.8 \mathrm{mmol})$, 1-(2-aminoethyl)pyrrolidine (102 $\mathrm{mg}, 0.9 \mathrm{mmol})$ and $\mathrm{Al}(\mathrm{Me})_{3}(3.2 \mathrm{ml}, 1.6 \mathrm{mmol})$; reaction time: $18 \mathrm{~h}$; purified by flash chromatography [pet. ether/EtOAc (5:5)]; yield: $198 \mathrm{mg}$ (65\%): $152 \mathrm{mg}$ as dark-brown semi-solid.

Microwave irradiation: Compound $5 \mathbf{f}$ was prepared from 4 (250 mg, $0.8 \mathrm{mmol})$ and 1-(2-aminoethyl)pyrrolidine (102 $\mathrm{mg}, 0.9 \mathrm{mmol}), 30 \mathrm{~min}$; column purification [pet. ether/EtOAc(1:1)]; yield: $198 \mathrm{mg}$ (65\%) as dark-brown semi-solid. M.p. $=142-145{ }^{\circ} \mathrm{C} .{ }^{1} \mathrm{H}$ NMR (400 MHz, DMSO) $\delta 8.70(\mathrm{~d}, 1 \mathrm{H}), 8.62(\mathrm{~s}, 1 \mathrm{H})$, 7.68 (d, 1H), 7.59 (br, 1H), 7.38 (m, 1H), 7.21-7.29 (m, 3H), 7.09 (d, 2H), 4.21 (s, $2 \mathrm{H}), 3.62(\mathrm{t}, 2 \mathrm{H}), 2.75(\mathrm{t}, 2 \mathrm{H}), 2.61(\mathrm{t}, 4 \mathrm{H}), 1.81(\mathrm{t}, 4 \mathrm{H}) .{ }^{13} \mathrm{C} \mathrm{NMR}(400 \mathrm{MHz}$, DMSO) $\delta 158.9,157.1,155.9,150.6,146.0,134.7,133.7,132.8,128.9,128.3$, 127.4, 54.7, 54.0, 38.2, 32.5, 23.5. LC-MS (ESI) $m / z: 377.4(\mathrm{M}+1)$. Anal. calcd. for $\mathrm{C}_{21} \mathrm{H}_{24} \mathrm{~N}_{6} \mathrm{O}$; C: 67.00; H: 6.43; N: 22.32. Found: C: 66.91; H: 6.30; N: 22.3.

\section{5-Benzyl-N-cyclobutyl-1-(pyridin-3-yl)-1H-1,2,4-triazole-3-carboxamide (5g)}

Conventional method: Compound 5g was prepared from 4 (250 mg, $0.8 \mathrm{mmol})$, cyclobutylamine $(64 \mathrm{mg}, 0.9 \mathrm{mmol})$ and $\mathrm{Al}(\mathrm{Me})_{3}(3.2 \mathrm{ml}, 1.6 \mathrm{mmol})$; reaction time: $16 \mathrm{~h}$; purified by flash column chromatography. [pet. ether/EtOAc (5:5)]; yield: $217 \mathrm{mg}(80 \%)$ as pale-yellow solid.

Microwave irradiation: Compound $5 \mathrm{~g}$ was prepared from 4 (250 mg, $0.8 \mathrm{mmol}$ ) and cyclobutylamine (64 mg, $0.8 \mathrm{mmol}), 30 \mathrm{~min}$; purified by flash column chromatography [pet. ether/EtOAc (1:1)]; yield: $237 \mathrm{mg}(88 \%)$ as pale-yellow solid. M.p. $=172-175{ }^{\circ} \mathrm{C} ;{ }^{1} \mathrm{H}$ NMR (400 MHz, DMSO) $\delta 8.71(\mathrm{~d}, 1 \mathrm{H}), 8.61(\mathrm{~s}$, 1H), $7.65(\mathrm{~d}, 1 \mathrm{H}), 7.39(\mathrm{~m}, 1 \mathrm{H}), 7.21-7.31(\mathrm{~m}, 4 \mathrm{H}), 7.09(\mathrm{~d}, 2 \mathrm{H}), 4.65(\mathrm{~m}, 1 \mathrm{H}), 4.21$ (s, 2H), $2.48(\mathrm{~m}, 2 \mathrm{H}), 2.05(\mathrm{~m}, 2 \mathrm{H}), 1.79(\mathrm{~m}, 2 \mathrm{H}) ;{ }^{13} \mathrm{C}$ NMR (400 MHz, DMSO) $\delta 158.9,157.1,155.9,150.6,146.0,134.7,133.7,132.8,128.9,128.3,127.4,54.7$, 
54.0, 38.2, 32.5, 23.5. LC-MS (ESI) $m / z: 377.4(\mathrm{M}+1)$. Anal. calcd. for $\mathrm{C}_{19} \mathrm{H}_{19} \mathrm{~N}_{5} \mathrm{O}$ :

C: 68.45; H: 5.74; N: 21.01. Found: C: 68.51; H: 5.65; N: 21.10.

\section{$N$-(2-Methoxybenzyl)-5-benzyl-1-(pyridin-3-yl)-1H-1,2,4-triazole-3-carboxamide} (5h)

Conventional method: Compound $\mathbf{5 h}$ was prepared from 4 (250 $\mathrm{mg}, 0.8 \mathrm{mmol})$, 2-methoxybenzylamine (123 $\mathrm{mg}, 0.9 \mathrm{mmol})$ and $\mathrm{Al}(\mathrm{Me})_{3}(3.2 \mathrm{ml}, 1.6 \mathrm{mmol})$; reaction time: $12 \mathrm{~h}$; purified by flash column chromatography [pet. ether/EtOAc (5:5)]; yield: $265 \mathrm{mg}(82 \%)$ as off-white solid.

Microwave irradiation: Compound $5 \mathrm{~h}$ was prepared from 4 (250 mg, $0.8 \mathrm{mmol})$ and 2-methoxybenzylamine $(123 \mathrm{mg}, 0.9 \mathrm{mmol}), 30 \mathrm{~min}$; purified by column chromatography [pet. ether/EtOAc (1:1)]; yield: $291 \mathrm{mg}(90 \%)$ as off-white solid. M.p. $=115-118{ }^{\circ} \mathrm{C} ;{ }^{1} \mathrm{H}$ NMR (400 MHz, DMSO) $\delta 8.71(\mathrm{~d}, 1 \mathrm{H}), 8.63(\mathrm{~s}, 1 \mathrm{H}), 7.65$ $(\mathrm{m}, 2 \mathrm{H}), 7.39(\mathrm{~m}, 2 \mathrm{H}), 7.31(\mathrm{~m}, 2 \mathrm{H}), 7.09(\mathrm{~d}, 2 \mathrm{H}), 6.91(\mathrm{~m}, 2 \mathrm{H}), 4.70(\mathrm{~d}, 2 \mathrm{H}), 4.19$ (s, 2H), 3.87 (s, 3H). ${ }^{13} \mathrm{C}$ NMR (400 MHz, DMSO) $\delta$ 150.6, 132.8, 129.9, 129.0, 128.9, 128.3, 127.4, 123.8, 120.7, 110.3, 55.3, 39.1, 32.5. LC-MS (ESI) $m / z: 400.1$ $(\mathrm{M}+1)$. Anal. calcd. for $\mathrm{C}_{23} \mathrm{H}_{21} \mathrm{~N}_{5} \mathrm{O}_{2}: \mathrm{C}: 69.16 ; \mathrm{H}: 5.30 ; \mathrm{N}: 17.53$. Found: $\mathrm{C}: 69.11$; $\mathrm{H}: 5.38 ; \mathrm{N}: 17.50$.

\section{Conclusions}

We have developed a simple and efficient method with excellent yields for synthesis of 1,2,4-triazole-3-carboxamides via one-pot synthesis, without using any other catalysts, in toluene. In this work, eight new compounds were successfully synthesized using both our reported method and the conventional method. The advantages of the present procedure are experimental simplicity, easy work-up procedure, mild reaction conditions and no requirement to use other catalysts. The yield of the present procedure is comparatively greater than for the conventional method (Table 1), not only that the unreacted starting materials were isolated from the present work.

Acknowledgments The authors express their profound gratitude to the VME Society, Islamiah College, Vaniyambadi for the laboratory facilities provided to carry out the research work. They also acknowledge the help rendered by Dr. Syed Shafi, Department of Chemistry, Thiruvalluvar University, Vellore. Authors are thankful to NMR Research Centre, Indian Institute of Science, Bangalore and CECRI, Karaikudi, TN for spectral studies.

Open Access This article is distributed under the terms of the Creative Commons Attribution License which permits any use, distribution, and reproduction in any medium, provided the original author(s) and the source are credited.

\section{References}

1. S. Bala, R.P. Gupta, M.L. Sachdeva, A. Singh, H.K. Pujari, Indian J. Chem. 16B, 481 (1978)

2. J. Mohan, Indian J. Chem. 22B, 270 (1983) 
3. A. Prasad, R.J. Ramalingam, A.B. Rao, P.V. Diwan, P.B. Sattur, Eur. J. Med. Chem. 24, 199 (1989)

4. A.H. El-masry, H.H. Fahmy, S.H. Ali Abdelwahed, Molecules 5, 1429 (2000)

5. A.S. Orabi, M.A. Moneim, E. El-Din Salem, M. El-Din Abdel-Fattah, Polish J. Chem. 74, 1675 (2000)

6. L.-Y. Wang, W.-C. Tseng, T.-S. Wu, K. Kaneko, H. Takayama, M. Kimura, W.-C. Yang, J.B. Wu, S.-H. Juang, F.F. Wong, Bioorg. Med. Chem. Lett. 21, 5358-5362 (2011)

7. G. Martin, German Patent, 2,240,043, March 1973. Chem. Abstr. 78, 136302 (1973)

8. S.S. Parmar, V.K. Rastogi, V.K. Agarwal, J.N. Sinha, A. Chaudhari, Can. J. Pharm. Soc. 9, 107 (1974)

9. T. George, D.V. Mehta, R. Tahilramani, J. Davvid, P.K. Talwalker, J. Med. Chem. 14, 335 (1971)

10. B. Dyck, V.S. Goodfellow, T. Phillips, J. Grey, M. Haddach, M. Rowbottom, G.S. Naeve, B. Brown, J. Saunders, Bioorg. Med. Chem. Lett. 14, 1151-1154 (2004)

11. J.H.M. Lange, H.H. Van Stuivenberg, H.K. Coolen, T.J. Adolfs, A.C. McCreary, H.G. Keizer, H.C. Wals, W. Veerman, A.J.M. Borst, W. De Looff, P.C. Verveer, C.G. Kruse, J. Med. Chem. 48, $1823-1838$ (2005)

12. J.A. Pfefferkorn, C. Choi, S.D. Larsen, B. Auerbach, R. Hutchings, W. Park, V. Askew, L. Dillon, J.C. Hanselman, Z. Lin, G.H. Lu, A. Robertson, C. Sekerke, M.S. Harris, A. Pavlovsky, G. Bainbridge, N. Caspers, M. Kowala, B.D. Tait, J. Med. Chem. 51, 31-45 (2008)

13. A. Benderly, S. Stavchansky, Tetrahedron Lett. 29, 739-740 (1988)

14. C.O. Kappe, Angew. Chem. Int. Ed. 43, 6250-6284 (2004)

15. P. Lidström, J. Tierney, B. Wathey, J. Westman, Tetrahedron 57, 9225-9283 (2001)

16. S. Caddick, Tetrahedron 51, 10403-10432 (1995) 